\title{
ANÁLISE DE SISTEMAS NÃO LINEARES INCERTOS: UMA ABORDAGEM LMI
}

\author{
Daniel Ferreira Coutinho ${ }^{\dagger *}$ \\ coutinho@das.ufsc.br \\ Alexandre Trofino* \\ trofino@das.ufsc.br \\ *Departamento de Automação e Sistemas, UFSC, Caixa Postal 476, CEP: 88040-900, Florianópolis, SC, Brasil. \\ ${ }^{\dagger}$ Licenciado do Departamento de Engenharia Elétrica, PUC-RS, Porto Alegre, RS, Brasil.
}

\begin{abstract}
In this paper, we address the problem of analysing the local stability of the equilibrium point of uncertain nonlinear systems and estimating its respective domain of attraction. We derive LMI conditions for the local robust stability problem based on Lyapunov functions which are polynomial functions of the state and uncertain parameters. The problem of maximizing the estimation of the domain of attraction consists of maximizing a level surface of the Lyapunov function inside a given polytope. Numerical examples show the performance of our approach.
\end{abstract}

KEYWORDS: Uncertain nonlinear systems, linear matrix inequalities, domain of attraction, polynomial Lyapunov functions.

\section{RESUMO}

Neste artigo, aborda-se o problema de análise de estabilidade local do ponto de equilíbrio de sistemas não lineares incertos e de seu respectivo domínio de atração. São propostas condições em termos de inequações matriciais lineares (LMIs) para o problema de estabilidade robusta local baseadas em funções de Lyapunov que são funções polinomiais dos estados e dos parâmetros incertos. O problema de maximização da estimativa do domínio de

Artigo submetido em $20 / 12 / 00$

1a. Revisão em 03/09/01

Aceito sob recomendação do Ed. Assoc. Prof. Liu Hsu atração consiste na maximização de uma curva de nível dentro de um dado politopo. Exemplos numéricos demonstram o desempenho desta metodologia.

PALAVRAS-CHAVE: Sistemas não lineares incertos, inequacoes matriciais lineares, domínio de atração, funções de Lyapunov polinomiais.

\section{INTRODUÇÃO}

Como determinar funções de Lyapunov é um problema fundamental em sistemas de controle. Muitos métodos têm sido propostos para obter obter tais funções no contexto de sistemas não lineares. Por exemplo, poderiamos mencionar as técnicas baseadas nas equações de Riccati (Mracek e Cloutier, 1998; Sznaier et al., 1998; Langson e Alleyne, 1999; Kazakova-Frehse e Moor, 1999) e na resolução de problemas convexos em termos de LMIs (El Ghaoui e Scorletti, 1996; Pettersson e Lennartson, 1997; Dussy e El Ghaoui, 1997; Kiriakidis, 1998; Johansen, 2000; Iwasaki, 2000).

Nestes métodos de determinação de funções de Lyapunov, é importante que a formulação permita uma fácil extensão para problemas de síntese ou de desempenho, como por exemplo $\mathcal{H}_{2}$ ou $\mathcal{H}_{\infty}$. Outra característica desejada é que a classe de sistemas abordados seja a mais abrangente possível.

Neste contexto, as técnicas LMI se mostram promissoras por solucionar uma grande variedade de problemas na teoria de controle com algorítimos numericamente efi- 
cientes. Em geral, os métodos propostos na literatura utilizam o conceito de estabilidade quadrática que aplicados a sistemas com incertezas conduzem a resultados conservadores, pois não levam em consideração a taxa de variação dos parâmetros. Por exemplo, o trabalho de (El Ghaoui e Scorletti, 1996) que utiliza funções de Lyapunov quadráticas apresenta resultados conservadores na análise de estabilidade de Sistemas Não Lineares Incertos.

Em (Trofino, 2000b) foi proposto um método de análise de estabilidade local de sistemas não lineares incertos, utilizando funções de Lyapunov polinomiais nos estados e parâmetros, na forma $v(x, \delta)=x^{\prime} \mathcal{P}(x, \delta) x$, introduzindo o conceito de estabilidade $\mathcal{Q}_{r}$.

Neste trabalho, que é a versão completa do artigo (Coutinho e Trofino, 2000), complementa-se o trabalho de (Trofino, 2000b) sobre a análise de estabilidade $\mathcal{Q}_{r}$, com as seguintes contribuições: $(i)$ realiza para o problema de estimativa da região de atração um estudo comparativo entre algumas abordagens encontradas na literatura; ( $i$ i) apresenta uma técnica para a estimativa da taxa de convergência das trajetórias do sistema; e (iii) extende os resultados para uma classe de sistemas com não linearidades trigonométricas.

\section{Notação}

Neste artigo, utiliza-se a notação usual. $I_{n}$ indica a matriz identidade de ordem $n \times n, 0_{n \times m}$ indica uma matriz de zeros de ordem $n \times m$ e $0_{n}$ indica uma matriz de zeros $n \times n . \quad P>0(\geq 0)$ significa que $P$ é uma matriz simétrica definida positiva (semi-definida positiva). $P^{\prime}$ é a matriz transposta de $P$. Sempre que possível as dimensões das matrizes são definidas pelo contexto. A derivada temporal da função $v(t)$ é simbolizada por $\dot{v}(t)$ onde o argumento $(t)$ será frequentemente omitido. $R_{A}$ representa a fronteira exata da região de atração de um ponto de equilíbrio e a região $\Upsilon$ é uma estimativa de $R_{A}$.

\section{FORMULAÇÃO DO PROBLEMA}

Seja a classe de sistemas não lineares incertos

$$
\dot{x}=A(x, \delta) x
$$

onde $x \in \mathbb{R}^{m_{0}}$ denota o vetor de estados, $\delta \in \mathbb{R}^{n_{\delta}}$ são os parâmetros incertos do sistema e $A(x, \delta)$ é uma função racional matricial de $(x, \delta)$. Assume-se que o lado direito da equação diferencial (1) é limitado para todos os valores de $x, \delta$ de interesse e $x=0$ é um ponto de equilíbrio.
Neste trabalho, estamos interessados em analisar as propriedades locais da origem do sistema (1) numa dada vizinhança representada por um dado politopo $\mathcal{B}_{x}$ : Assume-se que o parâmetro $\delta$ e a sua taxa de variação $\dot{\delta}$ não são conhecidos mas limitados por um dado politopo $\mathcal{B}_{\delta}$. A notação $(x, \delta, \dot{\delta}) \in \mathcal{B}_{x} \times \mathcal{B}_{\delta}$ implica que $x \in \mathcal{B}_{x}$ e $(\delta, \dot{\delta}) \in \mathcal{B}_{\delta}$

Para analisar a estabilidade do sistema (1) utilizaremos uma classe particular de funções de Lyapunov na caracterização da estabilidade exponencial. Para enfatizar esse ponto, apresentaremos a seguinte definição de estabilidade, proveniente de (Trofino, 2000b).

\section{Definição 2.1 (Estabilidade $\mathcal{Q}_{r}$ )}

Seja $\mathcal{B}=\mathcal{B}_{x} \times \mathcal{B}_{\delta}$ um dado meta-politopo, definindo uma vizinhança da origem e os valores admissíveis de $(\delta, \dot{\delta})$. A origem do sistema (1) é denominada localmente $\mathcal{Q}_{r}$ estável se existem funções de classe $\mathcal{K}, \phi_{1}(\cdot), \phi_{2}(\cdot), \phi_{3}(\cdot)$, e uma função do tipo $v(x, \delta)=x^{\prime} \mathcal{P}(x, \delta) x$, onde $\mathcal{P}(x, \delta)$ é uma matriz polinomial de grau " $\mathrm{r}$ " em $(x, \delta)$, tais que as seguintes condições estejam satisfeitas $\forall(x, \delta, \dot{\delta}) \in \mathcal{B}$ :

$$
\begin{aligned}
& \text { - } \phi_{1}(\|x\|) \leq v(x, \delta)=x^{\prime} \mathcal{P}(x, \delta) x \leq \phi_{2}(\|x\|) . \\
& \text { - } \dot{v}(x, \delta) \leq-\phi_{3}(\|x\|) .
\end{aligned}
$$

Em caso afirmativo, $v(x, \delta)=x^{\prime} \mathcal{P}(x, \delta) x$ é denominada de função de Lyapunov local para a origem.

Note que a noção de estabilidade $\mathcal{Q}_{r}$ implica na estabilidade exponencial da origem do sistema não linear incerto (Khalil, 1996) com um tipo particular de função de Lyapunov.

Para possibilitar uma formulação LMI do problema de análise de estabilidade considere que o sistema (1) possa ser reescrito na seguinte forma

$$
\left\{\begin{array}{l}
\dot{x}=A(x, \delta) x=\sum_{i=0}^{q} A_{i} \pi_{i}, \quad \Omega(x, \delta) \pi=0 \\
\pi_{0}=x, \quad \pi_{i+1}=\theta_{i} \pi_{i}, \quad i=\{0, \ldots, d-1\}
\end{array}\right.
$$

onde $x \in \mathbb{R}^{m_{0}}$ é o vetor de estados; $\delta \in \mathbb{R}^{n_{\delta}}$ são os parâmetros incertos do sistema; $\pi_{i} \in \mathbb{R}^{m_{i}}$ são variáveis auxiliares que permitem rescrever as não-linearidades do sistema, funções racionais de $(x, \delta)$, como uma função linear do vetor auxiliar $\pi=\left[\pi_{1}^{\prime} \ldots \pi_{q}^{\prime}\right]^{\prime} \in \mathbb{R}^{m}$ onde $m=\sum_{i=0}^{q} m_{i} ; A_{i} \in \mathbb{R}^{m_{0} \times m_{i}}$, para $i=\{0, \ldots, q\}$, são matrizes constantes associadas à dependência do sistema em relação às variáveis $\pi_{i} ; \theta_{i} \in \mathbb{R}^{m_{i+1} \times m_{i}}$, para $i=\{0, \ldots, d-1\}$, são funções matriciais afins em $(x, \delta)$ usadas para expressar as relações entre os vetores $\pi_{i}$. 
Note que, definidos dessa forma, $\pi_{i}$ é uma função polinomial de grau $i$ em $(x, \delta)$. A matriz $\Omega(x, \delta) \in \mathbb{R}^{m_{\Omega} \times m}$ é uma função matricial afim em $(x, \delta)$ usada para expressar relações adicionais entre os vetores $\pi_{i}$. Por exemplo, funções racionais em $(x, \delta)$ serão reescritas em termos das variáveis $\pi$ dessa forma.

Para simplificar a notação, não será mais explicitada a dependência das funções auxiliares $\pi_{i}, \theta_{i}$ e $\Omega$ em relação às variáveis $x, \delta$ e o tempo. Assume-se que o ponto de equilíbrio em análise é a origem do sistema $x=0$, e a representação do sistema em termos da variável $\pi$ é equivalente à representação (1), isto é, se a variável $\pi$ for eliminada se retorna à representação original (1).

Comentário 2.1 A classe de sistemas definida em (2) é mais abrangente do que as classes de sistemas polinomiais (Tibken, 2000) e engloba as representadas por frações lineares (LFR) (Dussy e El Ghaoui, 1997), comumente utilizadas em métodos que empregam LMIs, além disso é possível descrever vários tipos de não linearidades não racionais nesta classe de sistemas, veja para maiores detalhes a seção 6 .

Note, a partir da definição do sistema (2), que as matrizes $\theta_{i}$ são funções afins de $(x, \delta)$. Em particular, a matriz $\theta_{0}$, que como veremos a seguir tem um papel importante na construção da função de Lyapunov, pode ser representada na forma:

$$
\theta_{0}=\sum_{i=1}^{m_{0}} T_{i} x_{i}+\sum_{i=1}^{n_{\delta}} U_{i} \delta_{i}+V
$$

onde $T_{i}, U_{i}$ e $V$ são matrizes constantes tendo as mesmas dimensões de $\theta_{0}$ e $x_{i}, \delta_{i}$ são os i-ésimos termos dos vetores $x$ e $\delta$.

O próximo teorema apresenta condições suficientes, para o problema de análise de estabilidade, utilizando a definição de estabilidade $\mathcal{Q}_{2}$.

\section{Teorema 2.1 (Estabilidade $\mathcal{Q}_{2}$ )}

Considere o sistema não linear incerto (2), a representação (3) da matriz auxiliar $\theta_{0}$, seja $s_{i}$ a i-ésima linha da matriz identidade $I_{m_{0}}$ e a seguinte notação auxiliar:

$$
\begin{aligned}
\tilde{\theta}_{0} & =\sum_{i=1}^{m_{0}} T_{i} x s_{i} ; \quad \hat{\theta}_{0}=\sum_{i=1}^{n_{\delta}} U_{i} \dot{\delta}_{i} ; \\
A_{a} & =\left[\begin{array}{lr}
A_{0} & A_{1} \\
\hat{\theta}_{0}+\left(\theta_{0}+\tilde{\theta}_{0}\right) A_{0} & \left(\theta_{0}+\tilde{\theta}_{0}\right) A_{1}
\end{array}\right] ;
\end{aligned}
$$

$$
\begin{aligned}
A_{b}= & {\left[\begin{array}{ccccc}
A_{2} & & \cdots & & A_{q} \\
\left(\theta_{0}+\tilde{\theta}_{0}\right) A_{2} & \cdots & \left(\theta_{0}+\tilde{\theta}_{0}\right) A_{q}
\end{array}\right] ; } \\
C_{x}= & {\left[\begin{array}{ccccc}
x_{2} & -x_{1} & 0 & \cdots & 0 \\
0 & x_{3} & -x_{2} & \ddots & \vdots \\
\vdots & \ddots & \ddots & \ddots & 0 \\
0 & \cdots & 0 & x_{m_{0}} & -x_{m_{0}-1}
\end{array}\right] ; } \\
C_{a}= & {\left[\begin{array}{ccc}
C_{x} & 0 \\
\theta_{0} & -I_{m_{1}}
\end{array}\right] ; C_{b}=\left[\begin{array}{ccc}
\tilde{C}_{b} & 0
\end{array}\right] ; } \\
\tilde{C}_{b}= & {\left[\begin{array}{ccccc}
C_{x} & 0 & \cdots & \cdots & 0 \\
\theta_{0} & -I_{m_{1}} & \ddots & & \vdots \\
0 & \ddots & \ddots & \ddots & \vdots \\
\vdots & \ddots & \ddots & \ddots & \vdots \\
0 & \cdots & 0 & \theta_{d-1} & -I_{m_{d}}
\end{array}\right] . }
\end{aligned}
$$

onde $C_{x}$ é a matriz definida em (Trofino, 2000a), lema 2.1 .

Seja $\mathcal{B}_{x}$ um dado politopo definindo uma região na vizinhança da origem do sistema. Seja $\mathcal{B}_{\delta}$ um politopo definindo os valores admissíveis de $\delta$ e de sua taxa de variação $\dot{\delta}$. O sistema não linear incerto (2) é localmente $\mathcal{Q}_{2}$ estável se existem matrizes $P=P^{\prime}, L_{a}$ e $L_{b}$ com as mesmas dimensões de $A_{a}, C_{a}{ }^{\prime}$ e $C_{b}{ }^{\prime}$ respectivamente, tais que as seguintes LMIs sejam satisfeitas nos vértices do meta-politopo $\mathcal{B}_{x} \times \mathcal{B}_{\delta}$.

$$
\begin{aligned}
& P+L_{a} C_{a}+C_{a}^{\prime} L_{a}^{\prime}>0 \\
& {\left[\begin{array}{lr}
A_{a}^{\prime} P+P A_{a} & P A_{b} \\
A_{b}^{\prime} P & 0
\end{array}\right]+L_{b} C_{b}+C_{b}^{\prime} L_{b}^{\prime}<0}
\end{aligned}
$$

Em caso afirmativo, a função $v(x, \delta)=x^{\prime} \mathcal{P}(x, \delta) x$, com a matriz $\mathcal{P}(x, \delta)$ abaixo definida, é uma função de Lyapunov local para o sistema (2).

$$
\mathcal{P}(x, \delta)=\left[\begin{array}{c}
I_{m_{0}} \\
\theta_{0}
\end{array}\right]^{\prime} P\left[\begin{array}{c}
I_{m_{0}} \\
\theta_{0}
\end{array}\right]
$$

Observe que as condições propostas no teorema acima e no trabalho (Trofino, 2000b) não são equivalentes apesar de utilizarem a mesma noção de estabilidade.

\section{REGIÃO DE ATRAÇÃO}

Nesta seção, abordaremos o problema da determinação de uma estimativa adequada da região de atração do sistema não linear incerto (2). Para esse fim mostraremos que a região delimitada por uma certa curva de nível da função de Lyapunov se torna positivamente invariante se determinadas condições adicionais forem satisfeitas. A idéia é que a curva de nível deva estar contida no politopo $\mathcal{B}_{x}$ onde a função de Lyapunov é testada. Visando 
obter uma formulação convexa, faremos inicialmente algumas definições e considerações.

Por conveniência representaremos o politopo $\mathcal{B}_{x}$ pelos seus vértices, ou por um conjunto de inequações lineares na forma:

$$
\mathcal{B}_{x}=\left\{x: a_{k}^{\prime} x \leq 1, \quad k=1, \ldots, n_{f}\right\}
$$

onde $a_{k} \in \mathbb{R}^{m_{0}}$ são vetores dados associados as $n_{f}$ faces do politopo $\mathcal{B}_{x}$.

Desejamos encontrar condições para que a seguinte região seja uma estimativa da região de atração da origem do sistema (2).

$$
\Upsilon=\left\{x: v(x, \delta)=x^{\prime} \mathcal{P}(x, \delta) x \leq 1\right\}
$$

Observe que esta região não é um elipsóide usual, visto que $v(x, \delta)$ não é uma função quadrática em $x$.

Para garantirmos a invariância da região acima definida, devemos assegurar a seguinte relação de inclusão $\Upsilon \subset$ $\mathcal{B}_{x}$. A partir de (Boyd et al., 1994) e (Trofino, 2000a), a relação de inclusão é garantida se as seguintes LMIs

$$
\left[\begin{array}{cc}
1 & b_{k}^{\prime} \\
b_{k} & \left(P+L_{1} C_{a 1}+C_{a 1}^{\prime} L_{1}^{\prime}\right)
\end{array}\right] \geq 0, \quad \forall k
$$

são satisfeitas, para $k=\left\{1, \ldots, n_{f}\right\}$, nos vértices do politopo $\mathcal{B}_{x}$, onde os vetores auxiliares $b_{k}$ são dados por:

$$
b_{k}=\left[\begin{array}{c}
a_{k} \\
0
\end{array}\right] \in \mathbb{R}^{m_{0}+m_{1}}
$$

Resumindo, a factibilidade das LMIs (9) garante a relação de inclusão $\Upsilon \subset \mathcal{B}_{x}$ e a factibilidade de (4) garante $v(x, \delta)>0$ e $\dot{v}(x, \delta)<0$ ponto a ponto em $\mathcal{B}_{x}$. Logo a região (8) é positivamente invariante. Para a região $\Upsilon$ ser uma estimativa adequada da região de atração devemos maximizá-la em relaçao a algum índice, por exemplo em relação ao seu volume ou em relação ao semi-eixo maior, veja maiores detalhes em (Boyd et al., 1994). Neste trabalho, a maximização desta região será feita através do seguinte problema de otimização que deve ser resolvido nos vértices do meta-politopo $\mathcal{B}_{x} \times \mathcal{B}_{\delta}$.

$$
\min \lambda:\left\{\begin{array}{l}
(5),(9), \mathrm{e} \\
\lambda I-\left(P+L_{a} C_{a}+C_{a}{ }^{\prime} L_{a}{ }^{\prime}\right)>0
\end{array}\right.
$$

Exemplo 3.1 (Van der Pol em tempo reverso - VdPtr)

Aplicando a metodologia proposta no sistema Van der Pol em tempo reverso (Khalil, 1996), abaixo representado, podemos obter uma estimativa de $R_{A}$

$$
\left[\begin{array}{c}
\dot{x}_{1} \\
\dot{x}_{2}
\end{array}\right]=\left[\begin{array}{cc}
0 & -1 \\
1 & \epsilon\left(x_{1}^{2}-1\right)
\end{array}\right]\left[\begin{array}{l}
x_{1} \\
x_{2}
\end{array}\right]
$$

Estaremos assumindo que a constante de amortecimento não-linear é conhecida e dada por $\epsilon=1$.

Considere que o politopo $\mathcal{B}_{x}$ seja definido pelos seguintes vértices

$$
\left\{\left[\begin{array}{l}
\alpha \\
0
\end{array}\right],\left[\begin{array}{l}
0 \\
\alpha
\end{array}\right],\left[\begin{array}{c}
-\alpha \\
0
\end{array}\right],\left[\begin{array}{c}
0 \\
-\alpha
\end{array}\right]\right\}
$$

onde $\alpha$ é uma constante a ser determinada tal que $\mathcal{B}_{x}$ seja o maior possível. Note que neste sistema não temos incerteza, portanto $\mathcal{B}_{x} \times \mathcal{B}_{\delta}=\mathcal{B}_{x}$.

A figura 1 mostra os resultados para $\alpha=1.82$, considerando o problema de otimização (11) e as seguintes variáveis auxiliares

$$
\begin{aligned}
& \pi_{0}=\left[\begin{array}{ll}
x_{1} & x_{2}
\end{array}\right]^{\prime} ; \quad \pi_{1}=\left[\begin{array}{llll}
x_{1}^{2} & x_{1} x_{2} & x_{1} x_{2} & x_{2}^{2}
\end{array}\right]^{\prime} ; \\
& \pi_{2}=\left[\begin{array}{ll}
x_{1}^{3} & x_{1}^{2} x_{2}
\end{array}\right]^{\prime} ; \quad \theta_{0}=\left[\begin{array}{l}
x_{1} I_{2} \\
x_{2} I_{2}
\end{array}\right] ; \\
& \theta_{1}=\left[\begin{array}{ll}
x_{1} I_{2} & 0_{2}
\end{array}\right] ; \quad A_{0}=\left[\begin{array}{cc}
0 & -1 \\
1 & -1
\end{array}\right] ; \\
& A_{1}=\left[\begin{array}{cc}
0_{2} & 0_{2}
\end{array}\right] ; \quad A_{2}=\left[\begin{array}{cc}
0 & 0 \\
0 & 1
\end{array}\right] \text {; } \\
& \Omega=0 .
\end{aligned}
$$

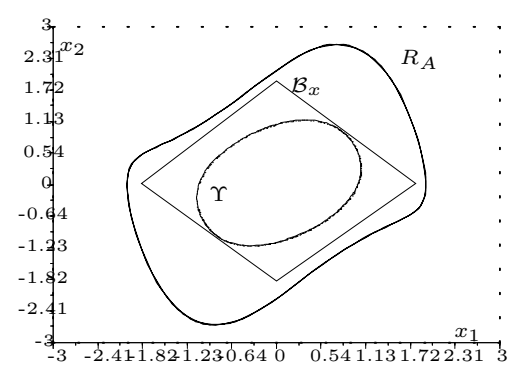

Figura 1: Região $R_{A}$, politopo $\mathcal{B}$ e estimativa $\Upsilon$

\section{Exemplo 3.2 (VdPtr incerto)}

Para ilustrar a aplicação da metodologia a sistemas incertos, considere que o coeficiente de amortecimento não linear do exemplo anterior seja um parametro incerto cujo valor é dado pela seguinte expressão:

$$
\epsilon=0.8+0.2 \delta
$$

onde $\delta \in[-1,1]$. Portanto, o sistema de VdPtr incerto pode ser escrito na seguinte forma:

$$
\left[\begin{array}{c}
\dot{x}_{1} \\
\dot{x}_{2}
\end{array}\right]=\left[\begin{array}{cc}
0 & -1 \\
1 & (0.8+0.2 \delta)\left(x_{1}^{2}-1\right)
\end{array}\right]\left[\begin{array}{l}
x_{1} \\
x_{2}
\end{array}\right]
$$


Considere a mesma definição utilizada no exemplo 3.1 para o politopo $\mathcal{B}_{x}$. Seja $\mathcal{B}_{\delta}$ o segmento de reta definido por $[-1,1]$.

A figura 2 mostra os resultados para $\alpha=1.45$, considerando o problema de otimização (11) e a seguinte formulação em termos das variáveis auxiliares

$$
\begin{aligned}
& \pi_{0}=\left[\begin{array}{l}
x_{1} \\
x_{2}
\end{array}\right] ; \quad \pi_{1}=\left[\begin{array}{lllll}
x_{1}^{2} & x_{1} x_{2} & x_{1} x_{2} & x_{2}^{2} & \delta x_{1}
\end{array}\right. \\
& \pi_{2}=\left[\begin{array}{c}
x_{1}^{3} \\
x_{1}^{2} x_{2}
\end{array}\right] ; \quad \pi_{3}=\delta x_{1}^{2} x_{2} ; \quad \theta_{0}=\left[\begin{array}{c}
x_{1} I_{2} \\
x_{2} I_{2} \\
\delta I_{2}
\end{array}\right] \text {; } \\
& \theta_{1}=\left[\begin{array}{ll}
x_{1} I_{2} & 0_{2 \times 4}
\end{array}\right] ; \quad \theta_{2}=\left[\begin{array}{cc}
0 & \delta
\end{array}\right] ; \quad \Omega=0 \\
& A_{0}=\left[\begin{array}{cc}
0 & -1 \\
1 & -0.8
\end{array}\right] ; \quad A_{1}=\left[\begin{array}{cc}
0_{1 \times 5} & 0 \\
01 \times 5 & -0.2
\end{array}\right] \text {; } \\
& A_{2}=\left[\begin{array}{cc}
0 & 0 \\
0 & 0.8
\end{array}\right] ; \quad A_{3}=\left[\begin{array}{c}
0 \\
0.8
\end{array}\right] \text {. }
\end{aligned}
$$

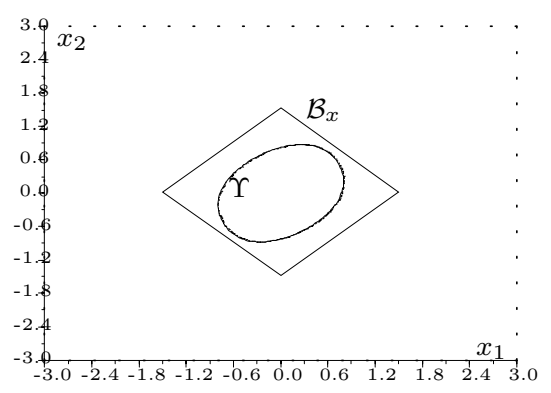

Figura 2: Politopo $\mathcal{B}_{x}$ e estimativa $\Upsilon$ para VdPtr com incerteza

Visando aprimorar a estimativa da região de atração analisaremos a influência da forma geométrica e do número de vértices do politopo $\mathcal{B}_{x}$, considerando o sistema do exemplo 3.1 (Van der Pol em tempo reverso sem incerteza).

No exemplo 3.1 utilizamos um politopo na forma de um losango simétrico em relação a origem. Pela forma geométrica estimada, podemos considerar um politopo $\mathcal{B}_{x}$ retangular com uma inclinação na direção do eixo maior da estimativa $\Upsilon$. A figura 3 apresenta a estimativa obtida para este politopo com as mesmas condições do exemplo 3.1.

Outro fator importante a considerar na melhoria da estimativa da região de atração é o número de vértices do politopo $\mathcal{B}_{x}$. Por exemplo, no caso do politopo da figura 3 aparentemente a estimativa $\Upsilon_{r}$ esta limitada na direção do seu eixo maior. A figura 4 mostra os resultados obtidos para um politopo $\mathcal{B}_{x}$ com 6 vértices considerando as mesmas condições do exemplo 3.1.

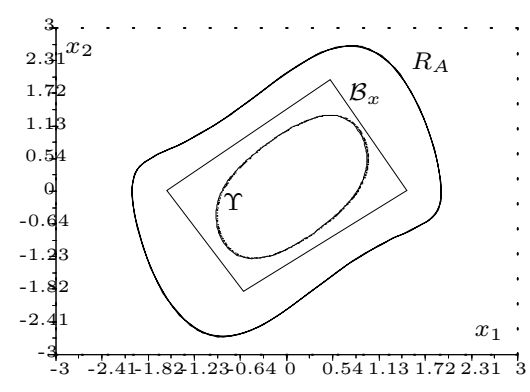

Figura 3: Região de atração $R_{A}$, politopo $\left(\mathcal{B}_{r}\right)$ e estimativa $\Upsilon$

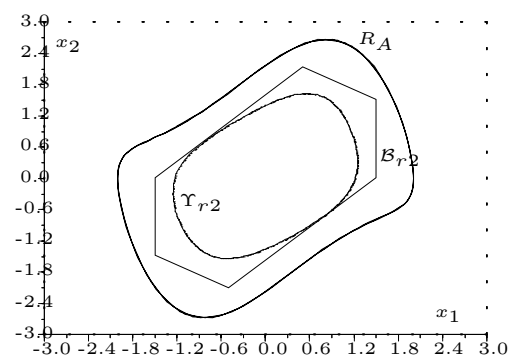

Figura 4: Região de Atração $R_{A}$, politopo $\mathcal{B}_{r 2}$ e estimativa $\Upsilon_{r 2}$

Analisando as figuras 3 e 4 verificamos que devemos explorar a forma do politopo $\mathcal{B}_{x}$ para aprimorarmos a estimativa da região de atração. Intuitivamente, já era esperado que o aumento do número de vértices melhoraria a estimativa da região de atração. Entretanto, devemos ter um certo critério na escolha dos vértices, já que, dependendo da situação, podemos piorar a estimativa. Outro fator a considerar, como veremos na sequência deste trabalho, é o aumento do esforço computacional gerado pelo aumento de vértices.

\section{ESTABILIDADE $\mathcal{Q}_{4}$}

Para aumentarmos o grau de liberdade na escolha da função de Lyapunov $v(x, \delta)=x^{\prime} \mathcal{P}(x, \delta) x$ podemos elevar a ordem da matriz $\mathcal{P}(x, \delta)$. Para tal, utilizaremos a noção de estabilidade $\mathcal{Q}_{4}$, onde a matriz $\mathcal{P}(x, \delta)$ é de grau 4 em relação a $(x, \delta)$.

O seguinte teorema propõe condições suficientes para estabilidade $\mathcal{Q}_{4}$ e pode ser visto como uma extensão do teorema 4.1 de (Trofino, 2000a), Para isso vamos considerar a seguinte notação para as matrizes $\theta_{0}$ e $\theta_{1}$, que por definição são afins em $(x, \delta)$.

$$
\theta_{i}=\sum_{j=1}^{m_{0}} T_{i j} x_{j}+\sum_{j=1}^{n_{\delta}} U_{i j} \delta_{j}+V_{i}, \quad i=\{0,1\}
$$


Teorema 4.1 (Estabilidade $\mathcal{Q}_{4}$ e região de atração)

Seja $\mathcal{B}$ um dado politopo, tal que $\left(x, \pi_{1}, \delta, \dot{\delta}\right) \in \mathcal{B}$. Considere o sistema (2), a representação (14) para as matrizes $\theta_{0}$ e $\theta_{1}$, a notação auxiliar (4) e

$$
\begin{aligned}
\tilde{\theta}_{i} & =\sum_{j=1}^{m_{0}} T_{i j} \pi_{i} s_{j} \text { e } \hat{\theta}_{i}=\sum_{j=1}^{n_{\delta}} U_{i j} \dot{\delta}_{j} \text { para } i=\{0,1\} \\
c_{k} & =\left[\begin{array}{cc}
a_{k}^{\prime} & 0
\end{array}\right]^{\prime} \in \mathbb{R}^{\left(m_{0}+m_{1}+m_{2}\right)} \\
\Gamma_{0} & =\hat{\theta}_{0}+\left(\theta_{0}+\tilde{\theta}_{0}\right) A_{0} \\
\Gamma_{1} & =\left(\theta_{0}+\tilde{\theta}_{0}\right) A_{1} \\
& \vdots \\
\Gamma_{q} & =\left(\theta_{0}+\tilde{\theta}_{0}\right) A_{q}
\end{aligned}
$$

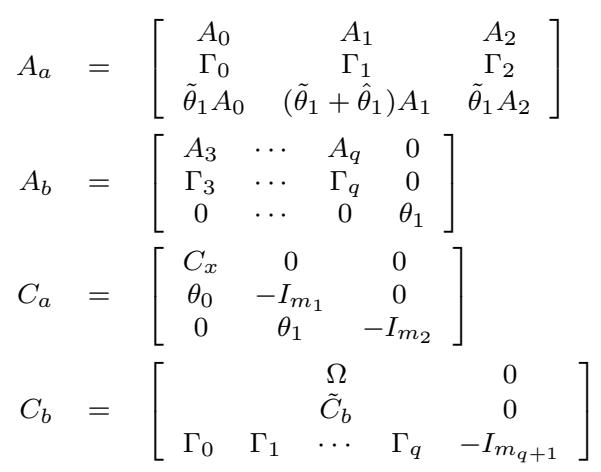

O sistema (2) é $\mathcal{Q}_{4}$ estável se existem matrizes $P=P^{\prime}$, $L_{a}$ e $L_{b}$, com as mesmas dimensões de $A_{a}, C_{a}^{\prime}$ e $C_{b}^{\prime}$, e um escalar $\lambda$, tais que as seguintes LMIs estejam satisfeitas nos vértices do politopo $\mathcal{B}$ :

$$
\begin{aligned}
& \min \quad \lambda \text { sujeito à } \\
& P+L_{a} C_{a}+C_{a}{ }^{\prime} L_{a}{ }^{\prime}>0 \\
& {\left[\begin{array}{cr}
\left(A_{a}{ }^{\prime} P+P A_{a}\right) & P A_{b} \\
A_{b}{ }^{\prime} P & 0
\end{array}\right]+L_{b} C_{b}+C_{b}{ }^{\prime} L_{b}{ }^{\prime}<0} \\
& {\left[\begin{array}{lr}
1 & c_{k}{ }^{\prime} \\
c_{k} & \left(P+L_{a} C_{a}+C_{a}{ }^{\prime} L_{a}{ }^{\prime}\right)
\end{array}\right] \geq 0, \quad \forall k} \\
& \lambda I-\left(P+L_{a} C_{a}+C_{a}{ }^{\prime} L_{a}{ }^{\prime}\right) \geq 0
\end{aligned}
$$

Em caso afirmativo, a função $v(x, \delta)=x^{\prime} \mathcal{P}(x, \delta) x$, com a matriz $\mathcal{P}(x, \delta)$ definida a seguir, é uma função de Lyapunov local e $\Upsilon=\left\{x: x^{\prime} \mathcal{P}(x, \delta) x \leq 1\right\}$ é uma estimativa da região de atração da origem do sistema (2).

$$
\mathcal{P}(x, \delta)=\left[\begin{array}{c}
I_{m_{0}} \\
\theta_{0} \\
\theta_{1} \theta_{0}
\end{array}\right]^{\prime} P\left[\begin{array}{c}
I_{m_{0}} \\
\theta_{0} \\
\theta_{1} \theta_{0}
\end{array}\right]
$$

O próximo exemplo ilustra a aplicação do teorema 4.1 e permite uma análise do compromisso entre o esforço computacional e o conservadorismo dos resultados.
Exemplo 4.1 Podemos obter uma estimativa da região de atração do sistema Van der Pol em tempo reverso utilizando a noção de estabilidade $\mathcal{Q}_{4}$ para as mesmas condições do exemplo (3.1). Para tal, consideraremos que o politopo $\mathcal{B}_{x}$ seja definido pelos seguintes vértices:

$\left\{\left[\begin{array}{c}-\alpha \\ -4 \alpha\end{array}\right],\left[\begin{array}{c}3 \alpha \\ 0\end{array}\right],\left[\begin{array}{c}3 \alpha \\ 3 \alpha\end{array}\right],\left[\begin{array}{c}\alpha \\ 4 \alpha\end{array}\right],\left[\begin{array}{c}-3 \alpha \\ 0\end{array}\right],\left[\begin{array}{l}-3 \alpha \\ -3 \alpha\end{array}\right]\right\}$

onde $\alpha$ é uma constante a ser determinada tal que $\mathcal{B}_{x}$ seja o maior possível. Aplicando o teorema 4.1, obtemse a matrix $P$ dada por (18).

A figura (5) mostra diversas estimativas do domínio de atração considerando os seguintes resultados: $\Upsilon_{a}$ (El Ghaoui e Scorletti, 1996); $\Upsilon_{b}$ (Davison e Kurak, 1971); $\Upsilon_{c}\left(\mathcal{Q}_{2}\right.$, com $\left.\alpha=0.53\right)$ e $\Upsilon_{d}\left(\mathcal{Q}_{4}, \operatorname{com} \alpha=0.60\right)$.

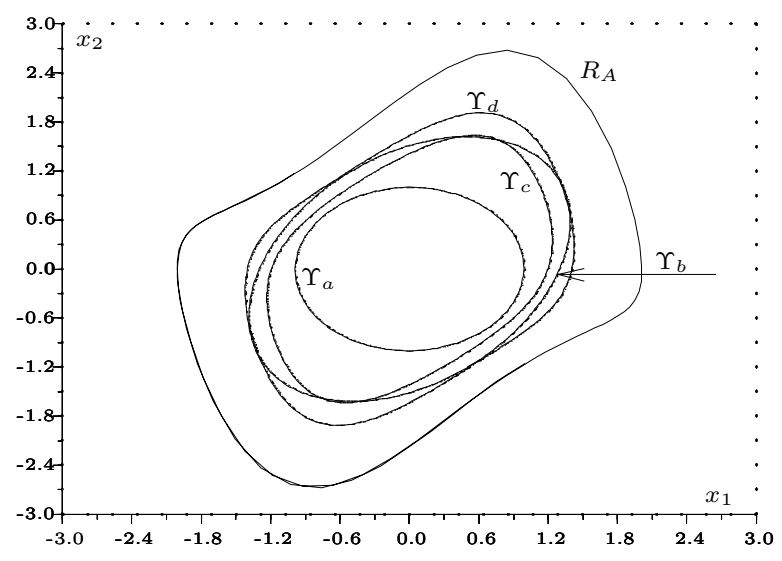

Figura 5: Diversas estimativas de $R_{A}$.

Verificamos que o aumento da ordem de $\mathcal{P}(x, \delta)$ produziu uma aproximação da Região de Atração da ordem de grandeza da proposta em (Davison e Kurak, 1971) e melhor do que a de (El Ghaoui e Scorletti, 1996). Poderíamos tentar melhorá-la colocando novos vértices nos extremos das direções $a_{1}=\left[\begin{array}{ll}1 & 1\end{array}\right]^{\prime}$ e $a_{2}=\left[\begin{array}{ll}1 & -1\end{array}\right]^{\prime}$. Em contrapartida, o aumento do número de vértices e o conceito de estabilidade $\mathcal{Q}_{4}$ produzem um grande aumento do esforço computacional.

A tabela 1 compara o esforço computacional para resolver o exemplo $3.1 \mathrm{com}$ as noções de estabilidade $\mathcal{Q}_{2}$ e $\mathcal{Q}_{4}$. A coluna NE representa a noção de estabilidade, NV o número de vértices, NL o número de LMIs e VD o número de variáveis de decisão.

\section{TAXA DE CONVERGÊNCIA}

Utilizando o princípio da invariância e o conceito de estabilidade exponencial, como em (Slotine e Li, 1991), 


$P=\left[\begin{array}{rrrrrrrr}0.54670 & -0.23060 & 0.00000 & -0.00001 & 0.00000 & -0.09229 & 0.00000 & 0.00811 \\ -0.23060 & 0.3420 & 0.00001 & 0.47732 & -0.38502 & 0.00000 & 0.10197 & -0.00072 \\ 0.00000 & 0.00000 & -0.08711 & -0.05815 & 0.01447 & 0.00544 & 0.00000 & 0.16394 \\ -0.00001 & 0.47732 & -0.05815 & 1.96711 & -1.36825 & -0.47213 & 0.00000 & -0.07157 \\ 0.00000 & -0.38502 & 0.01447 & -1.36825 & 0.83333 & 0.44604 & -0.16394 & 0.07157 \\ -0.09229 & 0.00000 & 0.00544 & -0.47213 & 0.44604 & 0.01179 & 0.00000 & 0.00000 \\ 0.00000 & 0.10197 & 0.00000 & 0.00000 & -0.16394 & 0.00000 & 0.03398 & 0.00236 \\ 0.00811 & -0.00072 & 0.16394 & -0.07157 & 0.07157 & 0.00000 & 0.00236 & -0.00013\end{array}\right]$

\begin{tabular}{|c|c|c|c|}
\hline NE & NV & VD & NL \\
\hline $\mathcal{Q}_{2}$ & 4 & 107 & 14 \\
\hline $\mathcal{Q}_{4}$ & 4 & 224 & 14 \\
\hline $\mathcal{Q}_{2}$ & 6 & 107 & 21 \\
\hline $\mathcal{Q}_{4}$ & 6 & 224 & 21 \\
\hline
\end{tabular}

Tabela 1: Esforço computacional $\times$ noção de estabilidade

podemos obter estimativas da região de atração e da taxa de convergência dos estados. É interessante notar que a estabilidade exponencial implica em estabilidade assintótica e fornece uma noção da localização dos estados em qualquer instante de tempo, similar ao conceito de pólos em sistemas lineares.

Considere que a função de Lyapunov $v(x, \delta)=$ $x^{\prime} \mathcal{P}(x, \delta) x$ e sua derivada são limitadas da seguinte maneira:

$$
\begin{aligned}
\left.\underline{\lambda}\|x\|_{2}^{2} \leq \quad x^{\prime} \mathcal{P}(x, \delta)\right) x & \leq \bar{\lambda}\|x\|_{2}^{2} \\
\dot{v}(x, \delta) & \leq-\varepsilon\|x\|_{2}^{2}
\end{aligned}
$$

onde $\bar{\lambda}, \underline{\lambda}$ e $\varepsilon$ são constantes positivas.

Suponha que as trajetórias do sistema (2) satisfaçam a seguinte relação

$$
\|x(t)\| \leq \beta\|x(0)\| e^{-\rho t}, \quad \forall x(0) \in \Upsilon \subseteq \mathcal{B}_{x}
$$

onde as constantes $\rho$ (conhecida como taxa de convergência) e $\beta$ são positivas.

Então, podemos fazer as seguintes considerações:

$$
\begin{aligned}
\underline{\lambda}\|x(t)\|^{2} & \leq v(x, \delta) \leq \bar{\lambda}\|x(t)\|^{2} \\
\underline{\lambda}\|x(0)\|^{2} & \leq v(0) \leq \bar{\lambda}\|x(0)\|^{2} \\
v(x, \delta) & \leq \alpha v(0) e^{-t / \eta}
\end{aligned}
$$

onde $\alpha=\frac{\beta^{2} \bar{\lambda}^{2}}{\underline{\lambda}^{2}}$ e $\eta=\frac{1}{2 \rho}$.

Utilizando o lema de convergência de (Slotine e Li, 1991), relacionamos $\eta$ com $v(x, \delta)$ e $\dot{v}(x, \delta)$ da seguinte forma

$$
\eta \dot{v}(x, \delta)+v(x, \delta) \leq 0
$$

O interesse da expressão acima é que ela pode ser expressa tem termos de LMIs. Note-se que a constante $\eta$ está relacionada com a taxa de convergência $\rho=\frac{1}{2 \eta}$ que é uma garantia da rapidez com que os estados convergem para a origem. Quanto menor $\eta$ mais rápido os estados convergem para a origem.

Com o mesmo procedimento da secção 4, podemos obter uma formulação LMI para o problema de estimar a região de atração com minimização da variável $\eta$.

Teorema 5.1 (Taxa de Convergência)

Seja $\mathcal{B}$ um dado politopo, tal que $\left(x, \pi_{1}, \delta, \dot{\delta}\right) \in \mathcal{B}$.

Considere o sistema (2) e as notações auxiliares (4), (15).

O sistema (2) é $\mathcal{Q}_{4}$ estável se existem matrizes $P=$ $P^{\prime}, L_{a}, L_{b}$ e uma constante $\eta>0$ tais que o seguinte problema de otimização esteja satisfeito nos vértices do politopo $\mathcal{B}$.

$$
\begin{aligned}
& \min \quad \eta \quad \text { sujeito a } \\
& P+L_{a} C_{a}+C_{a}{ }^{\prime} L_{a}{ }^{\prime}>0 \\
& \eta \times\left[\begin{array}{rr}
\left(A_{a}{ }^{\prime} P+P A_{a}\right) & P A_{b} \\
A_{b}{ }^{\prime} P & 0
\end{array}\right] \\
& \quad+\left[\begin{array}{rr}
\left(P+L_{a} C_{a}+C_{a}{ }^{\prime} L_{a}{ }^{\prime}\right) & 0 \\
0 & 0
\end{array}\right] \\
& \quad+L_{b} C_{b}+C_{b}{ }^{\prime} L_{b}{ }^{\prime}<0 \\
& {\left[\begin{array}{lrr}
1 & c_{k}{ }^{\prime} \\
c_{k} & \left(P+L_{a} C_{a}+C_{a} L_{a}{ }^{\prime}\right)
\end{array}\right]>0 \quad \forall k}
\end{aligned}
$$

Em caso afirmativo, a função $v(x, \delta)=x^{\prime} \mathcal{P}(x, \delta) x$, onde a matriz $\mathcal{P}(x, \delta)$ é dada por (17), é uma função de Lyapunov local, $\Upsilon=\left\{x: x^{\prime} \mathcal{P}(x, \delta) x \leq 1\right\}$ é uma estimativa da região de atração da origem do sistema (2) e a taxa de convergência satisfaz $\rho \geq \frac{1}{2 \eta}$.

Note que o teorema 5.1 foi formulado utilizando o conceito de estabilidade $\mathcal{Q}_{4}$, mas também pode ser aplicado ao conceito de estabilidade $\mathcal{Q}_{2}$. Para tal, basta zerarmos partes específicas da matriz $\mathcal{P}(x, \delta)$, veja (Trofino, 2000b) para maiores detalhes.

Exemplo 5.1 (Taxa de Convergência) Para exemplificar a aplicação do teorema 5.1 utilizaremos o sistema 
Van der Pol em tempo reverso. A figura 6 mostra os resultados para as mesmas condições do exemplo 3.1.

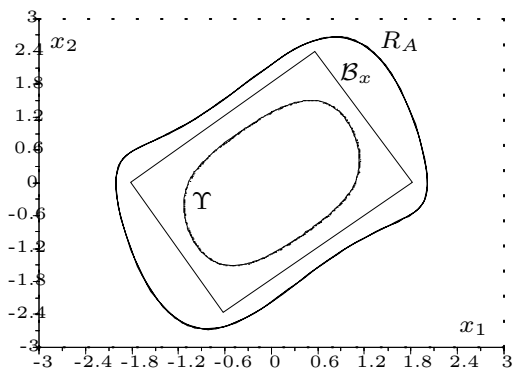

Figura 6: Estimativa $\Upsilon$ para $\rho \geq \frac{1}{76}$, considerando um politopo $\mathcal{B}_{r}$

O valor obtido para $\eta=38$ indica que a taxa de convergência satisfaz $\rho \geq \frac{1}{76}$. Isto é, as trajetórias do sistema (12) satisfazem a seguinte relação:

$$
\|x(t)\| \leq \beta\|x(0)\| e^{-t / 76}, \quad \forall x(0) \in \Upsilon
$$

\section{FUNÇÕES TRIGONOMÉTRICAS}

Com base no trabalho de (Trofino, 2000b), podemos obter uma formulação do tipo (2) para sistemas que possuam não linearidades trigonométricas. Devido à sua simplicidade a metodologia será apresentada diretamente no clássico exemplo do pêndulo.

Considere a representação por variáveis de estado do pêndulo apresentada em (Slotine e Li, 1991)

$$
\begin{aligned}
& \dot{x}_{1}=x_{2} \\
& \dot{x}_{2}=\alpha_{1} \sin x_{1}+\alpha_{2} x_{2}
\end{aligned}
$$

podemos definir duas variáveis auxiliares $x_{3}=\sin x_{1} \mathrm{e}$ $z=\cos x_{1}$. Aplicando em (24), obtemos duas novas relações:

- Diferencial: $\dot{x}_{3}=x_{2} z$;

- Algébrica: $x_{3}{ }^{2}+z^{2}=1$.

Portanto, podemos construir o seguinte sistema auxiliar:

$$
\dot{x}=\left[\begin{array}{ccc}
0 & 1 & 0 \\
0 & \alpha_{2} & \alpha_{1} \\
0 & z & 0
\end{array}\right] x, \forall(x, z): x_{3}{ }^{2}+z^{2}-1=0
$$

Para as condições iniciais $x_{1}(0), x_{2}(0), x_{3}(0)=$ $\sin \left(x_{1}(0)\right)$, as trajetórias do sistema do sistema original (24) se repetem no sistema auxiliar (25).
A escolha das matrizes $\theta_{i}$ da representação do sistema (2) não é única e influencia nos resultados obtidos. Portanto podemos explorar esta característica para melhorar a estimativa da região de atração do sistema (24).

Após vários testes, optamos pela seguinte formulação do sistema (25).

$$
\begin{aligned}
& \theta_{0}=\left[\begin{array}{ccc}
0 & 0 & x_{1} \\
z & 0 & 0 \\
0 & x_{2} & 0 \\
0 & 0 & x_{2} \\
0 & z & 0
\end{array}\right]=T_{1} x_{1}+T_{2} x_{2}+T_{z} z ; \quad \hat{\theta}_{0}=0 \\
& \tilde{\theta}_{0}=T_{1}\left[\begin{array}{lll}
x & 0 & 0
\end{array}\right]+T_{2}\left[\begin{array}{ccc}
0 & x & 0
\end{array}\right] ; \\
& \Omega=\left[\begin{array}{llllllll}
-1 & 0 & 0 & x_{3} & z & 0 & 0 & 0
\end{array}\right] .
\end{aligned}
$$

Pela formulação acima, o sistema auxiliar (25) é reescrito na forma: $\dot{x}=A_{0} \pi_{0}+A_{1} \pi_{1}, \forall x: \Omega \pi=0$, onde $\pi_{0}=\left[\begin{array}{lll}x_{1} & x_{2} & x_{3}\end{array}\right]^{\prime}$ e $\pi_{1}=\theta_{0} \pi_{0}$. Como neste caso a matriz $\theta_{0}$ depende da variável algébrica $z$, fato não previsto em (14), a matriz $A_{a}$ da notação auxiliar (4) sofre uma pequena modificação:

$$
A_{a}=\left[\begin{array}{lr}
A_{0} & A_{1} \\
\left(\theta_{0}+\tilde{\theta}_{0}\right) A_{0}+\hat{\theta}_{0} & \left(\theta_{0}+\tilde{\theta}_{0}\right) A_{1}+\theta_{z}
\end{array}\right]
$$

onde $\theta_{z}=T_{z}\left[\begin{array}{lllll}0 & 0 & 0 & -x & 0\end{array}\right]$.

A justificativa formal da mudança está nos apêndices.

A figura 7 mostra os resultados da aplicação do problema de otimização (11), com a matriz $A_{a}$ dada por (27), para o pêndulo considerando $\alpha_{1}=-1$ e $\alpha_{2}=-1$, a formulação equivalente (25), a notação auxiliar (26) e o conceito de estabilidade $\mathcal{Q}_{2}$.

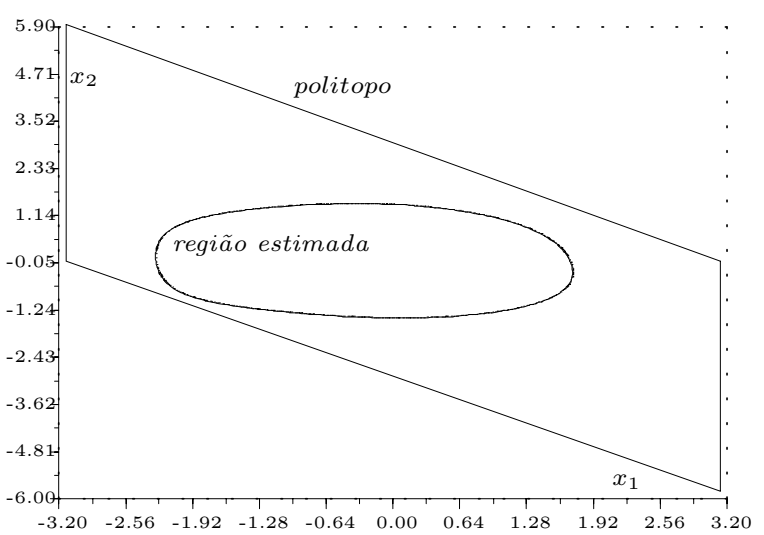

Figura 7: Estimativa da região de atração para o pêndulo considerando a noção de estabilidade $\mathcal{Q}_{2}$

\section{CONSIDERAÇÕES FINAIS}

Neste trabalho, apresentou-se uma técnica para análise de sistemas não lineares incertos, utilizando funções de 
Lyapunov polinomiais em relação aos estados e às incertezas do sistema. A classe de sistemas é bastante genérica permitindo a aplicação em sistemas com não linearidades racionais e algumas não racionais. Outra vantagem da metodologia proposta é a possibilidade de extensão desta aboradagem para problemas de síntese de controladores com custo garantido, veja para maiores detalhes (Coutinho et al., 2001).

Como principais contribuições, podemos citar o estudo de técnicas de maximização da estimativa da região de atração, aprimorando os resultados apresentados em (Trofino, 2000a) e (Trofino, 2000b), e a determinação de um limitante inferior da taxa de convergência do sistema. Também pode-se destacar a apresentação de estudos de casos, Van der Pol em tempo reverso (sem e com incerteza) e pêndulo. Neste último mostrou-se como incluir não linearidades trigonométricas na classe de sistemas proposta tratando do problema de determinação de uma estimativa da região de atração. Atualmente, estuda-se a extensão desta abordagem para problemas de síntese $\mathcal{H}_{\infty}$.

\section{AGRADECIMENTOS}

Os autores agradecem as contribuições fornecidas pelos revisores anônimos que colaboraram no aprimoramento deste trabalho. Este trabalho foi financiado pelo 'Conselho Nacional de Desenvolvimento Científico e Tecnológico - CNPq', sob processo número 147055/99-7 para o primeiro autor e número 300459/93-9/PQ para o segundo. Os exemplos numéricos foram obtidos através do software Scilab, (Gomez, 1999).

\section{REFERÊNCIAS}

Boyd, S., Ghaoui, L. E., Feron, E. e Balakrishnan, V. (1994). Linear Matrix Inequalities in Systems and Control Theory, SIAM books.

Coutinho, D. F. e Trofino, A. (2000). Análise de Sistemas Não Lineares Incertos: uma abordagem LMI, Proceedings of the XIII Congresso Brasileiro de Automática, Florianópolis.

Coutinho, D. F., Trofino, A. e Fu, M. (2001). Guaranteed Cost Control of Uncertain Nonlinear Systems via Polynomial Lyapunov Functions, Proceedings of the 40th IEEE Conference on Decision and Control, Orlando.

Davison, E. J. e Kurak, E. M. (1971). A Computational Method for Determining Quadratic Lyapunov Functions for Non-linear Systems, Automatica 1(7): $627-636$.
Dussy, S. e El Ghaoui, L. (1997). Multi-objective Bounded Control of Uncertain Nonlinear Systems: an Inverted Pendulum Example, Lecture Notes in Control and Information Sciences (n.227), SpringerVerlag.

El Ghaoui, L. e Scorletti, G. (1996). Control of Rational Systems using Linear-fractional Representations and LMIs, Automatica 32(9): 1273-1284.

Gomez, E. (1999). Engineering and Scientific Computing with Scilab, Birkhauser.

Iwasaki, T. (2000). Generalized Quadratic Lyapunov Functions for Nonlinear/Uncertain Systems Analysis, Proceedings of the 39th IEEE Conference on Decison and Control, Vol. 3, Sydney, pp. 29532958.

Johansen, T. A. (2000). Computation of Lyapunov functions for Smooth Nonlinear Systems using Convex Optimization, Automatica 36: 1617-1626.

Kazakova-Frehse, N. e Moor, T. (1999). Piecewise Constant Lyapunov Functions for the Construction of Robust Regions of Attraction, International Journal of Robust and Nonlinear Control 9: 451-460.

Khalil, H. K. (1996). Nonlinear Systems, Prentice Hall.

Kiriakidis, K. (1998). An LMI Approach to the Control of a Class of Nonlinear Systems, Proceedings of the 37th IEEE Conference on Decision and Control, Vol. 2, Tampa, pp. 1470-1475.

Langson, W. e Alleyne, A. (1999). A Stability Result with Application to Nonlinear Regulation: Theory and Experiments, Proceedings of the American Control Conference, Vol. 5, San Diego, pp. 30513056 .

Mracek, C. P. e Cloutier, J. R. (1998). Control Designs for the Nonlinear Benchmark Problem via the State-dependent Riccati Equation Method, International Journal of Robust and Nonlinear Control 8: 401-433.

Pettersson, S. e Lennartson, B. (1997). Exponential Stability Analysis of Nonlinear Systems using LMIs, Proceedings of the 36th IEEE Conference on Decision and Control, Vol. 1, San Diego, pp. 199-204.

Slotine, J.-J. e Li, W. (1991). Applied Nonlinear Control, Prentice Hall.

Sznaier, M., Cloutier, J., Hull, R., Jacques, D. e Mracek, C. (1998). A Receding Horizon State Dependent 
Riccati Equation Approach to SubOptimal Regulation of Nonlinear Systems, Proceedings of the 37th IEEE Conference on Decision and Control, Vol. 2, Tampa, pp. 1792-1797.

Tibken, B. (2000). Estimation of the Domain of Attraction for Polynomial Systems via LMI's, Proceedings of the 39th IEEE Conference on Decision and Control, Vol. 4, Sydney, pp. 3860-3864.

Trofino, A. (2000a). Bi-quadratic stability of uncertain nonlinear systems, Proceedings of the 3rd IFAC Symposium on Robust Control Design, Prague.

Trofino, A. (2000b). Robust Stability and Domain of Attraction of Uncertain Nonlinear Systems, Proceedings of the American Control Conference, Vol. 5, Chicago, pp. 3707-3711.

\section{Prova do Teorema 2.1}

Visando simplificar a prova, vamos considerar a seguinte particição do vetor auxiliar $\pi$,

$$
\pi=\left[\begin{array}{c}
\pi_{a} \\
\pi_{b}
\end{array}\right] \quad: \quad \pi_{a}=\left[\begin{array}{c}
\pi_{0} \\
\pi_{1}
\end{array}\right] \quad \text { e } \quad \pi_{b}=\left[\begin{array}{c}
\pi_{2} \\
\vdots \\
\pi_{q}
\end{array}\right]
$$

onde $\pi_{a} \in \mathbb{R}^{m_{a}}\left(m_{a}=m_{0}+m_{1}\right), \pi_{b} \in \mathbb{R}^{m_{b}}\left(m_{b}=\right.$ $\left.m_{2}+\cdots+m_{q}\right)$, e representar a primeira LMI de (5) por $M_{1}>0$ e a segunda por $M_{2}<0$.

Suponha que as duas condições de (5) estejam satisfeitas nos vértices do politopo $\mathcal{B}=\mathcal{B}_{x} \times \mathcal{B}_{\delta}$, então, por convexidade, estão satisfeitas para $\forall(x, \delta, \dot{\delta}) \in \mathcal{B}$.

Seja $Y_{a} \in \mathbb{R}^{m_{0} \times m_{a}}$ uma matrix tal que $Y_{a} \pi_{a}=x$ e defina $Y_{b}=\left[\begin{array}{ll}Y_{a} & 0_{m_{0} \times m_{b}}\end{array}\right]$. Observe que por construção $C_{x} x=0$ e $\theta_{0} x-\pi_{1}=0$, portanto $C_{a} \pi_{a}=0$.

Como a primeira LMI de (5) é estrita $\left(M_{1}>0\right)$, para um escalar positivo $\varepsilon_{1}$ suficientemente pequeno, é possível adicionar o termo $-\varepsilon_{1} Y_{a}^{\prime} Y_{a}$ sem que isso mude o sinal da desigualdade, isto é, $M_{1}-\varepsilon_{1} Y_{a}^{\prime} Y_{a} \geq 0$. Pré e pós multiplicando esta condição por $\pi_{a}^{\prime}$ e $\pi_{a}$ respectivamente, pode-se reescreve-la da seguinte forma

$$
\pi_{a}^{\prime} P \pi_{a}=x^{\prime} \mathcal{P}(x, \delta) x \geq \varepsilon_{1} x^{\prime} x, \quad \forall(x, \delta, \dot{\delta}) \in \mathcal{B}
$$

Visto que $x, \delta, \dot{\delta}$ pertencem a um politopo, os elementos de $M_{1}$ são limitados. Então existe um escalar positivo $\varepsilon_{2}$, suficientemente grande tal que $\varepsilon_{2} I_{m_{a}, \geq M_{1}}$. Logo $\varepsilon_{2} \pi_{a}^{\prime} \pi_{a} \geq \pi_{a}^{\prime} M_{1} \pi_{a}$ que leva a $\varepsilon_{2}\left(x^{\prime} x+x^{\prime} \theta_{0}^{\prime} \bar{\theta}_{0} x\right) \geq$ $x^{\prime} \mathcal{P}(x, \delta) x$. Lembrando que $x, \delta, \dot{\delta}$ pertencem a um politopo, existe um escalar positivo $\varepsilon_{3}$ suficientemente grande tal que $\varepsilon_{3} I_{m_{a}} \geq \theta_{0}^{\prime} \theta_{0}$. Portanto

$$
x^{\prime} \mathcal{P}(x, \delta) x \leq \varepsilon_{2}\left(1+\varepsilon_{3}\right) x^{\prime} x, \quad \forall(x, \delta, \dot{\delta}) \in \mathcal{B}
$$

Considere a segunda LMI de (5). Como ela é estrita $\left(M_{2}<0\right)$, para um escalar positivo $\varepsilon_{4}$ suficientemente pequeno, é possível adicionar o termo $\varepsilon_{4} Y_{b}^{\prime} Y_{b}$ tal que $M_{2}+\varepsilon_{4} Y_{b}^{\prime} Y_{b} \leq 0$ é satisfeita. Note que por construção $C_{x} x=0, \theta_{i} \pi_{i}-\pi_{i+1}=0(i=0, \ldots, d-1)$ e $\Omega \pi=$ 0 , portanto $C_{b} \pi=0$. Pré e pós multiplicando $M_{2} \leq$ $-\varepsilon_{4} Y_{b}^{\prime} Y_{b}$ por $\pi^{\prime}$ e $\pi$ respectivamente, pode-se reescrever esta inequação matricial da seguinte maneira

$$
\pi_{a}^{\prime}\left(A_{a}^{\prime} P+P A_{a}\right) \pi_{a}+2 \pi_{a}^{\prime} P A_{b} \pi_{b} \leq-\varepsilon_{4} x^{\prime} x, \quad \forall(x, \delta, \dot{\delta}) \in \mathcal{B}
$$

Utilizando as seguintes igualdades

$$
\begin{aligned}
\pi_{a}^{\prime} P A_{a} \pi_{a} & =x^{\prime} \mathcal{P}(x, \delta) x\left[\begin{array}{ll}
A_{0} & A_{1}
\end{array}\right] \pi_{a} \\
& +x^{\prime}\left[\begin{array}{c}
I_{m_{0}} \\
\theta_{0}
\end{array}\right]^{\prime} P\left[\begin{array}{c}
0 \\
\dot{\theta}_{0}
\end{array}\right] x, \mathrm{e} \\
\pi_{a}{ }^{\prime} P A_{b} \pi_{b} & =x^{\prime} \mathcal{P}(x, \delta)\left[\begin{array}{lll}
A_{2} & \cdots & A_{q}
\end{array}\right] \pi_{b}
\end{aligned}
$$

mostra-se que $\dot{v}(x, \delta)=\pi_{a}^{\prime}\left(A_{a}^{\prime} P+P A_{a}\right) \pi_{a}+2 \pi_{a}^{\prime} P A_{b} \pi_{b}$. Portanto

$$
\dot{v}(x, \delta) \leq-\varepsilon_{4} x^{\prime} x
$$

Finalizando, as condições (29), (30) e (31) mostram que a função $v(x, \delta)=x^{\prime} \mathcal{P}(x, \delta) x$ é uma função de Lyapunov local e que a origem do sistema (2) é $\mathcal{Q}_{2}$ estável.

\section{Prova do Teorema 4.1}

Suponha que as LMIs de (16) estejam satisfeitas nos vértices do politopo $\mathcal{B}$. Por convexidade, também estão para $\forall\left(x, \pi_{1}, \delta, \dot{\delta}\right) \in \mathcal{B}$.

Definindo uma variável auxiliar $\pi_{q+1}=\dot{\pi}_{1}$ e considerando o vetor $\pi=\left[\begin{array}{cc}\pi_{a}{ }^{\prime} & \pi_{b}{ }^{\prime}\end{array}\right]^{\prime}$, onde

$$
\pi_{a}=\left[\begin{array}{c}
\pi_{0} \\
\pi_{1} \\
\pi_{2}
\end{array}\right] \text { e } \quad \pi_{b}=\left[\begin{array}{c}
\pi_{3} \\
\vdots \\
\pi_{q} \\
\pi_{q+1}
\end{array}\right]
$$

Observa-se que:

- $\dot{\pi}_{a}=A_{a} \pi_{a}+A_{b} \pi_{b}$ 


\section{- $\pi_{q+1}=\left[\begin{array}{llll}\Gamma_{0} & \cdots & \Gamma_{q} & 0\end{array}\right] \pi$}

E a prova segue de forma idêntica a do teorema 2.1.

\section{Prova do Teorema 5.1}

Defina as constantes $\bar{\lambda}$ e $\underline{\lambda}$ como

$$
\begin{aligned}
& \bar{\lambda}=\min _{(x, \delta)} \quad \lambda:\left\{\lambda I_{m_{a}}-\left(P+L_{a} C_{a}+C_{a}^{\prime} L_{a}^{\prime}\right) \geq 0\right\} \\
& \underline{\lambda}=\max _{(x, \delta)} \quad \lambda:\left\{P+L_{a} C_{a}+C_{a}^{\prime} L_{a}^{\prime}-\lambda I_{m_{a}} \geq 0\right\}
\end{aligned}
$$

Observe que $\underline{\lambda}, \bar{\lambda}$ correspondem respectivamente aos auto-valores mínimo e máximo, em $(x, \delta)$, da matriz $\left(P+L_{a} C_{a}+C_{a}^{\prime} L_{a}^{\prime}\right)$.

Seja a partição (28) do vetor $\pi$. Note que da mesma maneira que na prova do teorema 2.1, por construção tem-se que $C_{a} \pi_{a}=0$ e $C_{b} \pi=0$.

Suponha que a condição 5.1 esteja satisfeita nos vértices do politopo $\mathcal{B}, \operatorname{logo}$, por convexidade, também está satisfeita para $\forall x \in \mathcal{B}_{x}$ e $\forall(\delta, \dot{\delta}) \in \mathcal{B}_{\delta}$.

Considere a primeira LMI de (23), aplicando a definição (32) podemos reescreve-la como:

$$
\underline{\lambda} I_{m_{a}} \leq\left(P+L_{a} C_{a}+C_{a}^{\prime} L_{a}^{\prime}\right) \leq \bar{\lambda} I_{m_{a}}
$$

Pré e pós multiplicando a expressão acima por $\pi_{a}^{\prime}$ e seu transposto, tem-se que:

$$
\underline{\lambda} \pi_{a}^{\prime} \pi_{a} \leq \pi_{a}^{\prime}\left(P+L_{a} C_{a}+C_{a}^{\prime} L_{a}^{\prime}\right) \pi_{a} \leq \bar{\lambda} \pi_{a}^{\prime} \pi_{a}
$$

Pela definição de estabilidade $\mathcal{Q}_{r}$, tem-se que $v(x, \delta)=$ $x^{\prime} \mathcal{P}(x, \delta) x=\pi_{a}^{\prime} P \pi_{a}$. Logo a expressão acima é equivalente a:

$$
\underline{\lambda}\left\|\pi_{a}\right\|^{2} \leq v(x, \delta) \leq \bar{\lambda}\left\|\pi_{a}\right\|^{2}
$$

Considere a segunda LMI de (23). Pré e pós multiplicando-a por $\pi^{\prime}$ e seu transposto, tem-se que:

$$
\eta\left(\dot{\pi}_{a}^{\prime} P \pi_{a}+\pi_{a}^{\prime} P \dot{\pi}_{a}\right)+\pi_{a}^{\prime} P \pi_{a}<0
$$

Visto que $\dot{v}(x, \delta)=\dot{\pi}_{a}^{\prime} P \pi_{a}+\pi_{a}^{\prime} P \dot{\pi}_{a}$, a condição acima corresponde a:

$$
\eta \dot{v}(x, \delta)+v(x, \delta)<0
$$

Por (33) e (34) o sistema (2) é $\mathcal{Q}_{r}$ estável. Além disso, $v(x, \delta)$ satistaz:

$v(x(t), \delta(t)) \leq v(x(0), \delta(0)) e^{-t / \eta}, \quad \forall x \in \mathcal{B}_{x}, \quad(\delta, \dot{\delta}) \in \mathcal{B}_{\delta}$

Observe que $\left\|\pi_{a}\right\| \geq\|x\|$, a partir de (33) a expressão acima pode ser reescrita como:

$$
\|x(t)\|^{2} \leq \frac{\bar{\lambda}}{\underline{\lambda}}\left\|\pi_{a}(0)\right\|^{2} e^{-t / \eta}, \quad \forall x \in \mathcal{B}_{x},(\delta, \dot{\delta}) \in \mathcal{B}_{\delta}
$$

Note que $\left\|\pi_{a}(0)\right\|$ é uma constante finita limitada pelo politopo $\mathcal{B}$.

Pela definição (3.5) de (Khalil, 1996) a expressão (35) implica na estabilidade exponencial do sistema (2), onde um limitante inferior da taxa de convergência é dado por $\rho \geq \frac{1}{2 \eta}$.

Finalizando, a terceira LMI corresponde à relação de inclusão $\Upsilon \subset \mathcal{B}_{x}$, completando a prova.

\section{PROVA DO TEOREMA 2.1 COM VARIÁ- VEL ALGÉBRICA}

Seja $z \in \mathbb{R}$ uma variável algébrica do sistema (2). Suponha que a matriz $\theta_{0}$ seja uma função afim em $x, \delta, z$. Logo pode-se representar a matriz $\theta_{0}$ da seguinte maneira

$$
\theta_{0}=\sum_{i=1}^{m_{0}} T_{i} x_{i}+\sum_{i=1}^{n_{\delta}} U_{i} \delta_{i}+T_{z} z+V
$$

onde as matrizes $T_{i}, U_{i}, V$ e os escalares $x_{i}, \delta_{i}$ são com em (3) e $T_{z}$ é uma matriz constante com as mesmas dimensões de $\theta_{0}$.

Na determinação da derivada temporal de $v(x, \delta)$ aparecerá o termo $\dot{\theta}_{0} x=\tilde{\theta}_{0} \dot{x}+\hat{\theta}_{0} x+T_{z} x \dot{z}$. Sem perda de generalidade, considere que $\dot{z}$ é uma das componentes do vetor $\pi_{1}$. Defina um vetor $N_{z} \in \mathbb{R}^{m_{1}}$ tal que $N_{z}^{\prime} \pi_{1}=\dot{z}$. Portanto, pode-se redefinir a matriz $A_{a}$ em (4) da seguinte maneira

$$
A_{a}=\left[\begin{array}{cc}
A_{0} & A_{1} \\
\left(\theta_{0}+\tilde{\theta}_{0}\right) A_{0}+\hat{\theta}_{0} & \left(\theta_{0}+\tilde{\theta}_{0}\right) A_{1}+\theta_{z}
\end{array}\right]
$$

onde $\theta_{z}=T_{z} x N_{z}^{\prime}$.

E a prova segue de forma idêntica a do teorema 2.1. 